Case Report: Open Access

\title{
Polycythemia Vera with High Erythropoietin Level: Case Report
}

\author{
Rami Nassabein ${ }^{1 *}$, Mariam al khatib ${ }^{2}$, Marie Helen Abbas ${ }^{3}$, Marie youssef $^{2}$, Daniel Amer ${ }^{4}$ \\ and Jaafar Wazni ${ }^{3}$
}

\author{
${ }^{1}$ Hematology Oncology Department, Rafic Hariri University Hosipital, Lebanese University, Lebanon \\ 2Internal Medicine Department, Rafic Hariri University Hosipital, Lebanese University, Lebanon \\ ${ }^{3}$ Neurology Department, Rafic Hariri University Hosipital, Lebanese University, Lebanon \\ ${ }^{4}$ Department of Surgery, Rafic Hariri University hospital, Beirut Arab University, Lebanon
}

${ }^{*}$ Corresponding author: Rami Nassabein, MD, Hematology-Oncology fellow at Rafic Hariri University hospital, Lebanese University, Bahaa Building- $5^{\text {th }}$ floor-closed street near BIG SALE shopping center-Barbir-Beirut-Lebanon, Tel:009613064454, E-mail: rami.nassabein@hotmail.com

\begin{abstract}
Polycythemia Vera (PV) is a myeloproliferative disorder of bone marrow stem cells characterized by erythrocytosis. Elevated erythropoietin level strongly indicates secondary erythrocytosis and excludes PV diagnosis. However, patients with PV who co-present with Budd-Chiari syndrome (BCS) have been documented with elevated serum erythropoietin levels provided that JAK2 mutation is positive.
\end{abstract}

\section{Keywords}

Polycythemia vera, Erythropoietin, Budd-Chiari syndrome

\section{Introduction}

In the presence of symptoms, Erythrocytosis, warrants further investigation to distinguish primary causes from secondary. It is well established that high erythropoietin levels exclude primary causes of $\mathrm{PV}$, which requires searching for secondary causes. In place this leads us to the following:

Can high erythropoietin level be present in PV?

\section{Case Report}

A married male of 49-years-old, with maternal family history of hypertension and dyslipidemia, was presented to our hospital. $\mathrm{He}$ complained of a one week duration severe headache, with no focal deficit on neurological exam. Brain CT without contrast was done, to show no evidence of intracranial bleed. Labs showed Hemoglobin of $18 \mathrm{~g} \backslash \mathrm{dl}$, platelet count of 156 and WBC of $12300 / \mathrm{mm}^{3}$. Due to the above results, the patient was admitted for further investigation.

Evaluation of secondary causes of erythrocytosis was started. Erythropoietin level was $161.8 \mathrm{mIU} / \mathrm{ml}$ (NL: 2.6-18.5 mIU/ml). Brain magnetic resonance imaging/arteriography and venography revealed no thrombosis or brain tumor. The patient is a non-smoker, with no evidence of hypoxia by ABG's and lives at sea level. CT scan of the abdomen and pelvis was done for the developing jaundice. However, no evidence of renal vessel narrowing, nor kidney or liver mass were shown. Alpha fetoprotein level $1.8 \mathrm{ng} \backslash \mathrm{ml}$.
The laboratory results showed: INR: 2.8 corrected with mixing studies, LFTs: SGOT 51.9IU/L, SGPT: 49 IU/L, GGT: 363 IU/L, alkaline phosphatase: $158 \mathrm{IU} / \mathrm{L}$, direct bilirubin $9.8 \mathrm{mg} / \mathrm{dl}$ with negative hepatitis profile. CT scan of the abdomen and pelvis that was done earlier has revealed cirrhotic liver features, large splenomegaly with significant varices around the stomach cardia, splenic helium and perisplenic area, the intrahepatic portal vein is patent, but the extrahepatic part is poorly visualized together with the splenic vein suggestive of partial thromobosis, with mild ascites.

The patient was still suffering from severe pain even though he was given a multiple course of pain killers (acetaminophen, NSAID, Morphine). Therefore; a phlebotomy was ordered; hence, the removal of one unit of RBC's, leading to pain relief.

Due to the accumulation of significant amount of ascites, he suffered an increase in his abdominal girth. Abdominal paracentesis was performed with a serum ascetic albumin gradient of 2.2 suggestive transudate. Due to these outcomes with the CT findings, liver biopsy and magnetic resonance venography (MRV) of the abdomen were made. MRV of the abdomen revealed: "Dysmorphic feature with enlarges segment I and atrophy of its right posterior sector. No focal lesion, the hepatic veins are small and thin leading to a highly suggestion of Budd-Chiari Syndrome (BCS)." Liver biopsy showed, "A chronic hepatitis with mild piecemeal necrosis and lobular necrosis, with moderate portal expansion and bridging fibrosis observed. Metavir Score: A1 F2."

Since no clear cause of secondary erythrocytosis was revealed; bone marrow aspirate and biopsy were performed. It showed erythroblastic proliferation, increase of mature and immature megakaryocytes, as well as an increase in polymorphs. Reaching panmyelosis, the picture was consistent with PV. Real time PCR for V617F mutation in the JAK2 gene was positive.

Patient was diagnosed with Budd-Chiari syndrome due to Polycythemia Vera on top of chronic liver disease. He was put on aspirin after gastroscopy with esophageal varices ligation. 


\section{Discussion}

The abnormal proliferation of $\mathrm{PV}$ is sustained by oncogenic mutations in JAK-STAT pathway (signal transducers and activators of transcription). These mutations are the V617F mutation within exon 14 (95\% of PV) and other different mutations within exon 12 ( $4 \%$ of PV) of the JAK2 gene [1].

Low erythropoietin is used in the diagnosis of $\mathrm{PV}$, as it is the minor diagnostic criteria according to WHO classification 2016 [2] In the presence of elevated serum erythropoietin levels, searching for secondary causes of polycythemia is attempted [3].

PV is one of the most frequent condition causing BCS (10-40\% of cases). In patients with polycythemia co-presenting with BCS, it has been described in few reports to have high serum Erythropoietin levels $(4,5$, and 6$)$. Thumures, et al. [4]; described four patients that were presented with BCS, with elevated red cell mass, and founded to have elevated serum erythropoietin level where polycythemia was confirmed with JAK2 mutation assay and bone marrow biopsy examination [5]. Jones $\mathrm{C}$, et al. [6] also described a patient that was presented with acute Budd-Chiari syndrome, and found to have elevated hemoglobin level, high serum erythropoietin level, and positive JAK2 mutation which was treated with rivaroxaban (xarelto).

It was hypothesized that Erythropoietin production is regulated in an oxygen-dependent manner, and driven via hypoxia-inducible transcription factors (HIF)-1 and (HIF)-2, that are believed to be upregulated in patients with BCS having hypoxic liver injury and hepatocyte necrosis [7].

Our patient was presented with severe headache, elevated HB level, hepatosplenomegaly, ascites, and found to have Budd-Chiari syndrome. Erythropoietin level was high, although bone marrow aspirate and biopsy where suggestive of PV. In addition to the two major principles of WHO criteria of diagnosis: JAK-2 mutation and elevated HB level. This can be explained by the postulated hypothesis that BCS caused hypoxia of the liver in the absence of clinical hypoxia, and lead to high erythropoietin levels. In such patient the identification of JAK 2 V617F mutation confirm the diagnosis of PV.

\section{Conclusion}

PV in the presence of Budd-Chiari syndrome can have elevated erythropoietin levels; hence, diagnosis is reached by identification of the JAK-2 mutation.

\section{References}

1. Passamonti F (2012) How I treat polycythemia vera. Blood 120: 275-284.

2. Arber DA, Orazi A, Hasserjian R, Thiele J, Borowitz MJ, et al. (2016) The 2016 revision to the World Health Organization classification of myeloid neoplasms and acute leukemia. Blood 127: 2391-2405.

3. Birgegård G, Wide L (1992) Serum erythropoietin in the diagnosis of polycythaemia and after phlebotomy treatment. $\mathrm{Br} \mathrm{J}$ Haematol 81: 603-606.

4. Thurmes PJ, Steensma DP (2006) Elevated serum erythropoietin levels in patients with Budd-Chiari syndrome secondary to Polycythemia Vera: Clinical implications for the role of JAK2 mutation analysis. Eur J Haematol 77: 57-60.

5. Levy VG, Ruskone A, Baillou C, Theirman-Duffaud D, Najman A, et al. (1985) Polycythemia and the Budd-Chiari syndrome: study of serum erythropoietin and bone marrow erythroid progenitors. Hepatology 5: 858-861.

6. Jones C, Levy Y, Tong AW (2014) Elevated serum erythropoietin in a patient with polycythaemia vera presenting with Budd-Chiari syndrome. BMJ Case Rep 2014.

7. Aydinli M, Bayraktar Y (2007) Budd-Chiari syndrome: etiology, pathogenesis and diagnosis. World J Gastroenterol 13: 2693-2696. 